\title{
Persistent disruption of ciliated epithelium following paediatric lung transplantation
}

\author{
Biju Thomas*, Paul Aurora\#, Helen Spencer", Martin Elliott" , Andrew Rutman*, \\ Robert A. Hirst* and Christopher O’Callaghan*,\#
}

ABSTRACT: It is unclear whether ciliary function following lung transplantation is normal or not. Our aim was to study the ciliary function and ultrastructure of epithelium above and below the airway anastomosis and the peripheral airway of children following lung transplantation.

We studied the ciliary beat frequency (CBF) and beat pattern, using high speed digital video imaging and ultrastructure by transmission electron microscopy, of bronchial epithelium from above and below the airway anastomosis and the peripheral airway of 10 cystic fibrosis (CF) and 10 non-suppurative lung disease (NSLD) paediatric lung transplant recipients.

Compared to epithelium below the anastomosis, the epithelium above the anastomosis in the CF group showed reduced CBF (median (interquartile range): 10.5 (9.0-11.4) Hz versus 7.4 (6.49.2) $\mathrm{Hz} ; \mathbf{p}<0.01)$ and increased dyskinesia (median (IQR): 16.5 (12.9-28.2)\% versus 42.2 (32.656.4)\%; $\mathbf{p}<0.01)$. In both CF and NSLD groups, compared with epithelium above the anastomosis, the epithelium below the anastomosis showed marked ultrastructural abnormalities (median duration post-transplant 7-12 months).

Ciliary dysfunction is a feature of native airway epithelium in paediatric CF lung transplant recipients. The epithelium below the airway anastomosis shows profound ultrastructural abnormalities in both CF and NSLD lung transplant recipients, many months after transplantation.

KEYWORDS: Bronchial epithelium, ciliary beat frequency, ciliary beat pattern, cystic fibrosis, nonsuppurative lung disease

ung transplantation is an accepted treatment option for children with end-stage lung disease. Compared with other solid organ transplants, the overall survival remains poor for paediatric lung transplant recipients [1]. According to recent reports [1], almost half the deaths within the first year post lung transplant are attributable to infection. Infections may also play a role in the pathogenesis of bronchiolitis obliterans syndrome (BOS), which is the major cause of death by 5 yrs post lung transplant [2-5].

The ciliated epithelium that covers the surface of the airways forms an immunologically active natural barrier to invasion and injury by inhaled pathogenic organisms. The epithelium is lined by the airway surface liquid in which the cilia beat at a frequency of 10-14 Hz, clearing the mucus with its trapped pathogens. This process, known as mucociliary clearance [6], is an essential factor in pulmonary defence. Impaired mucociliary clearance and respiratory epithelial damage lead to increased susceptibility to infection and inflammation.
Though available evidence from both animal $[7,8]$ and human $[9,10]$ studies suggests that the mucociliary clearance may be impaired in lung transplant recipients, particularly in the early posttransplant period, the reasons for this impairment are far from being completely understood. Limited evidence suggests possible epithelial ultrastructural abnormalities [10] and alterations in airway mucus rheology $[7,11]$ in the early post-operative period following lung transplantation. Though a small number of studies examined ciliary beat frequency $(\mathrm{CBF})$ in adult lung transplant recipients, the finding of a significantly reduced $\mathrm{CBF}$ in the transplanted bronchi compared to that of the native bronchi by some authors [12] could not be replicated by others $[9,13,14]$. To date, there have been no studies that examined ciliary function in children following lung transplantation and no previous paediatric or adult studies have evaluated ciliary beat pattern post lung transplantation. Assessment of ciliary beat pattern in addition to CBF has recently been recognised as an essential part in evaluating ciliary function, as cilia may
AFFILIATIONS

*Dept of Infection, Immunity and Inflammation, University of Leicester Leicester, and

\#Dept of Cardiothoracic

Transplantation, Great Ormond Street Hospital for Children, London, UK.

CORRESPONDENCE

C. O'Callaghan

Dept of Respiratory Medicine

Portex Unit

Institute of Child Health

University College London (UCL) and Great Ormond Street Hospital

30 Guilford Street

WC1N 1EH

London

UK

E-mail: ajb64@le.ac.uk

Received:

Oct 082011

Accepted after revision:

Jan 212012

First published online:

April 202012 
maintain their beat frequency whilst beating in a grossly dyskinetic fashion $[15,16]$.

The aim of this study was to assess the ciliary function and detailed ultrastructure of the respiratory epithelium proximal to the airway anastomosis and compare them to the airway distal to the airway anastomosis and that of the peripheral airway, in paediatric lung transplant recipients.

\section{METHODS}

\section{Subjects}

Over a 2-yr period (2007-2009) 20 children (aged <18 yrs), who underwent flexible bronchoscopy following lung transplantation at the Great Ormond Street Hospital for Children (London, UK), were studied and demographic and clinical details were collected. All subjects underwent $\mathrm{pH} /$ impedance studies to examine for evidence of pathological gastro-oesophageal reflux. Flexible bronchoscopy was conducted according to the European Respiratory Society guidelines [17]. Epithelial brushings were taken from $2-3 \mathrm{~cm}$ above and $2-3 \mathrm{~cm}$ below the airway anastomosis under direct vision and from a peripheral airway under fluoroscopic screening that confirmed the brush to be at the very edge of the lung in all cases; detailed methodology can be found in the online supplementary material. Bronchoalveolar lavage (BAL) was performed and the fluid was tested for various pathogens including bacteria, mycobacteria, fungi and viruses. BAL was also processed using the Oil Red $\mathrm{O}$ staining technique to estimate the proportion of lipid-laden macrophages and a proportion $>10 \%$ was considered significant. Transbronchial lung biopsy samples were obtained and histopathological studies were performed to look for evidence of rejection. At the time of collection of bronchial epithelial samples, the subjects had been free from intercurrent respiratory infections, for a minimum of 6 weeks.

The study protocol was approved by the Institute of Child Health and Great Ormond Street Hospital Research Ethics Committee. Participating children provided assent and written informed consent was obtained from parents.

\section{CBF and beat pattern}

Detailed methodology is given in the online supplementary material. CBF was measured and beat pattern assessed on strips of bronchial epithelium using a digital high-speed video microscopy system, as described previously with nasal epithelial brushings [18], within 4-6 h of sample collection. The high-speed video images were analysed in a blinded fashion. The images were re-analysed by a second observer (A. Rutman) and blinded on a second occasion by the original observer (B. Thomas).

The experimental system allowed the ciliary beat pattern to be evaluated in three different planes: a sideways profile, beating directly toward the observer, and beating from directly above [18]. The path taken by a cilium during the beat cycle was analysed frame by frame. This was characterised and compared with the normal beat pattern as seen on digital high-speed video analysis [18]. Dyskinesia was defined as an abnormal beat pattern that included: a reduced beat amplitude, a stiff beat pattern, the failure to bend along the length of the ciliary shaft, a flickering or twitching motion, and static cilia. Dyskinesia index was calculated as the percentage of dyskinetic cilia within the sample (number of dyskinetic readings/total number of readings for sample $\times 100)$. The immotility index was calculated as the percentage of immotile cilia within the sample (number of immotile readings/total number of readings for sample $\times 100$ ) [19]. A micrometre was used to calibrate and measure the length of cilia from the digital sideways images of ciliated epithelial edges projected onto a computer screen.

\section{Transmission electron microscopy}

Detailed ultrastructure of the bronchial epithelial strips was studied using transmission electron microscopy, as described before with nasal epithelial brushings [20]. Detailed methodology

\section{TABLE 1 Demographic and clinical details}

\begin{tabular}{|c|c|c|}
\hline & Cystic fibrosis & $\begin{array}{c}\text { Non-suppurative lung } \\
\text { disease }\end{array}$ \\
\hline Subjects & 10 & 10 \\
\hline Sex $M / F$ & $2 / 8$ & $7 / 3$ \\
\hline Age yrs & $14.1(12.8-15.7)$ & $13.1(8.6-15.4)$ \\
\hline \multicolumn{3}{|l|}{ Diagnosis } \\
\hline Cystic fibrosis & 10 & \\
\hline Obliterative bronchiolitis & & 3 \\
\hline $\begin{array}{l}\text { Pulmonary arterial } \\
\text { hypertension }\end{array}$ & & 4 \\
\hline $\begin{array}{l}\text { Pulmonary venoocclusive } \\
\text { disease }\end{array}$ & & 1 \\
\hline Interstitial lung disease & & 1 \\
\hline Eisenmenger syndrome & & 1 \\
\hline \multicolumn{3}{|l|}{ Type of transplant } \\
\hline Bilateral lung & 9 & 7 \\
\hline Heart-lung & 1 & 3 \\
\hline \multicolumn{3}{|l|}{ Indication for bronchoscopy } \\
\hline Surveillance & 9 & 9 \\
\hline Suspected rejection & 1 & 1 \\
\hline $\begin{array}{l}\text { Duration post-transplant } \\
\text { months }\end{array}$ & $12(2.9-15.5)$ & $7(2.3-15)$ \\
\hline $\begin{array}{l}\text { FEV }_{1} \% \text { pred at } \\
\text { time of bronchoscopy }\end{array}$ & $75.5(54.5-96)$ & $74(60.5-97)$ \\
\hline \multicolumn{3}{|l|}{ BAL } \\
\hline No organisms & 6 & 5 \\
\hline URT commensals & 3 & 4 \\
\hline Mycobacterium abscessus & 1 & \\
\hline Streptococcus pneumoniae & & 1 \\
\hline \multicolumn{3}{|l|}{ Transbronchial biopsy } \\
\hline No acute cellular rejection & 10 & 10 \\
\hline AOBO & 8 & 7 \\
\hline AOBX & 2 & 3 \\
\hline $\begin{array}{l}\text { Gastro-oesophageal reflux } \\
\text { disease requiring Nissen } \\
\text { fundoplication }\end{array}$ & 9 & 8 \\
\hline $\begin{array}{l}\text { BAL lipid laden } \\
\quad \text { macrophages }>10 \%\end{array}$ & 3 & 4 \\
\hline
\end{tabular}

Data are presented as $\mathrm{n}$ or median (interquartile range). M: male; $\mathrm{F}$ : female FEV1: forced expiratory volume in $1 \mathrm{~s} ; \%$ pred: $\%$ predicted; BAL: bronchoalveolar lavage; URT: upper respiratory tract; $A 0$ : no evidence of acute rejection; BO: no airway inflammation/lymphocytic bronchiolitis; BX: insufficient specimen for assessment. 

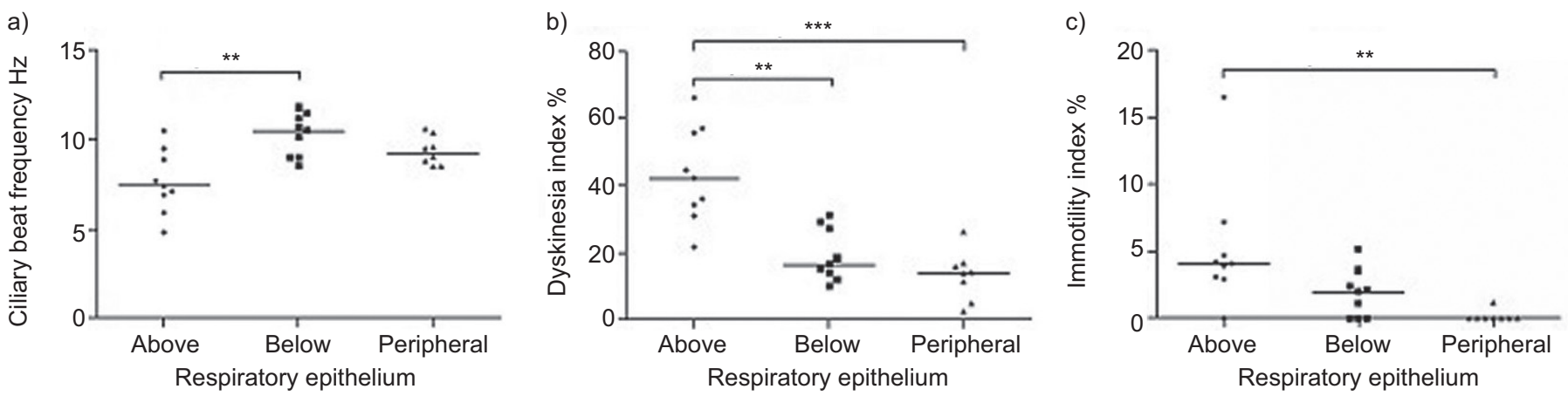

FIGURE 1. a) Ciliary beat frequency, b) dyskinesia index and c) immotility index of respiratory epithelium above and below the airway anastomosis and the peripheral airway of paediatric cystic fibrosis lung transplant recipients. ${ }^{* *}: p<0.01$; ${ }^{* *}$ : $p<0.001$.

is given in the online supplement. The ciliated epithelium was assessed, in a blinded fashion, for both epithelial and ciliary ultrastructural changes. The number of ciliated cells, unciliated cells, mucus cells, and dead cells were expressed as a percentage of all cells examined. Disruption and damage to the epithelium was assessed by calculating the percentages of ciliated cells with loss of cilia, cellular projections, cytoplasmic blebbing and mitochondrial damage, among all cells examined. Damage to individual cilium was evaluated by examining ciliary ultrastructure for microtubular and dynein arm defects and the percentage of cilia with microtubular or dynein arm defects was calculated. Intracellular ciliary orientation, defined as the standard deviation of the angles of lines through the central pair of microtubules of cilia originating from a single ciliated cell, was determined as described previously [21].

\section{Statistical analyses}

Sample size was calculated based on CBF as the primary outcome measure. SEYBOLD et al. [22] studied the surface liquid velocity on freshly excised sheep trachea and found that a $16 \%$ increase in CBF correlated with a 56\% increase in tracheal surface liquid velocity. Hence, we assumed that an absolute mean difference in $\mathrm{CBF}$ of $2 \mathrm{~Hz}$ has potential biological significance. It was estimated that, to detect a mean difference in CBF of $2 \mathrm{~Hz}$ (with a standard deviation of $1 \mathrm{~Hz}$ ) between two groups, with a confidence interval of $95 \%$ and a power of $80 \%$, a sample size of six in each group would be required. Statistical analysis was performed using GraphPad Prism 5. Non-parametric data were described as median (interquartile range (IQR)). Groups were initially compared using the Friedman test and post-hoc analysis was performed using Dunn's method. $\mathrm{p}<0.05$ was considered statistically significant. Agreement between the two observers (B. Thomas and A. Rutman) was excellent for measurement of CBF (interclass correlation 0.94) as well as dyskinesia index (interclass correlation 0.93). Repeatability (agreement within observer) was also excellent (interclass correlation was 0.94 for $\mathrm{CBF}$ and 0.99 for dyskinesia index).

\section{RESULTS}

The demographic and clinical details, including results of the $\mathrm{pH} /$ impedance study, BAL, and transbronchial biopsy, are given in table 1 . None of the transbronchial biopsy samples studied showed evidence of acute cellular rejection. Of the 20 children studied, two children, who had a pathogenic organism in the BAL, were excluded from the ciliary function studies and analysis of epithelial ultrastructure. $\mathrm{CBF}$ and beat pattern were studied in 18 children (nine each in the cystic fibrosis (CF) group and the non-suppurative lung disease (NSLD) group) the results are given in figures 1 and 2 and tables S1 and S2 in the online supplementary material.

In the CF group, the median (IQR) CBF was significantly decreased in the epithelium above the airway anastomosis (7.4 (6.4-9.2) Hz) compared with the epithelium below (10.5 (9.011.4) Hz; Friedman $\mathrm{p}<0.01$ ) (fig. 1a). Analysis of ciliary beat pattern showed a higher proportion of dyskinetic cilia in the epithelium above the airway anastomosis (42.2 (32.6-56.4)\%) compared with that below the anastomosis (16.5 (12.9-28.2)\%) and that of the peripheral airway (13.9 (6.5-16.6)\%; Friedman
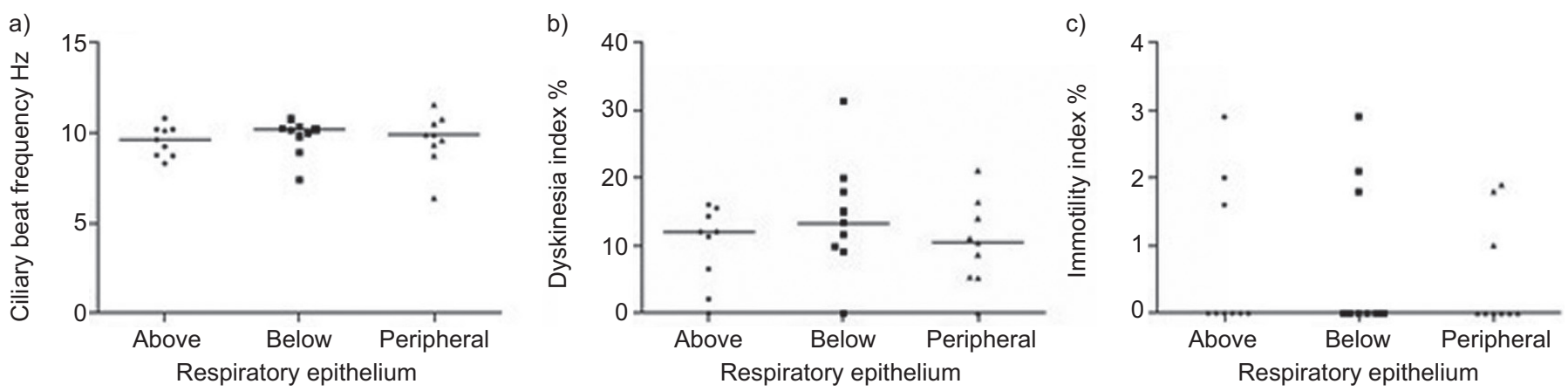

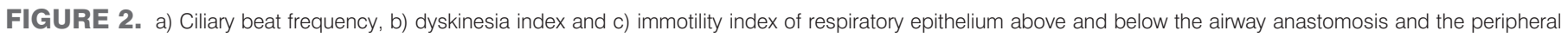
airway of paediatric non-suppurative lung disease lung transplant recipients. 
TABLE 2 Analysis of epithelial ultrastructure of the cystic fibrosis group by transmission electron microscopy

\begin{tabular}{lccc} 
& Above anastomosis & Below anastomosis & Peripheral airway \\
\hline Ciliated cells & & & \\
Unciliated cells & $67.8(64.1-73.9)^{\star *}$ & $36.9(34.9-39.1)$ & $67.7(55-71.1)^{\star}$ \\
Mucus cells & $25.5(20.8-30.4)^{\star *}$ & $45.1(42.9-46.1)$ & $28.1(19.7-35.4)$ \\
Dead cells & $5.5(4.6-6.7)$ & $6.4(5.7-6.9)$ & $6.9(4.9-7.2)$ \\
Dynein arm defects & $0^{\star *}$ & $11.7(10.9-13.1)$ & $1.2(0-2.3)$ \\
Microtubular defects & $2.9(1.1-5.2)$ & $3.6(0.4-4.2)$ & $3(2.6-4.5)$ \\
Ciliated cells with loss of cilia & $2.2(2-2.6)^{\star *}$ & $6.3(5.3-8.7)$ & $2.1(2.1-3.9)^{\star}$ \\
Cells extruding from the surface & $22.6(14.8-26.7)^{\star *}$ & $87.7(78.3-92.4)$ & $29.7(17.8-49.4)^{\star}$ \\
Cells with cytoplasmic blebbing & $20.9(14.1-25.5)^{\star *}$ & $83.8(77.2-89.7)$ & $28.1(25.5-31.3)$ \\
Cells with mitochondrial damage & $10.7(8.5-12.9)^{\star *}$ & $40.7(33.7-44.2)$ & $17.9(15.6-20.6)$ \\
Ciliary orientation degrees & $8.8(7.1-11.6)^{* *}$ & $34.3(26.5-39.8)$ & $16.2(13.5-20.4)$ \\
\hline
\end{tabular}

Data are presented as median percentage (interquartile range). *: $p<0.05 ;{ }^{*}: p<0.01$ when compared with epithelium below the airway anastomosis.

$\mathrm{p}<0.01$ ) (fig. 1b). The epithelium above the airway anastomosis also showed significantly higher immotility index (4.1 (3-5.9)\%) compared with that of the peripheral airway $(0 \%)$ (Friedman $\mathrm{p}<0.001$; fig. 1c). In contrast, in the NSLD group, there was no significant difference in the CBF, dyskinesia index or immotility index between the epithelium above and below the airway anastomosis and that of the peripheral airway (fig. 2). The median (IQR) length of cilia in the central (above and below the airway anastomosis) and peripheral airways were 5.8 (5.46.2) $\mu \mathrm{m}$ and 6.0 (5.6-6.2) $\mu \mathrm{m}$, respectively, and the difference was not statistically significant $(p=0.17)$.

Bronchial brushing obtained from one child each in the CF group and the NSLD group was excluded from analysis of epithelial ultrastructure because of identification of a pathogenic organism in the bronchoalveolar lavage fluid. The samples obtained from two other children each in the CF and the NSLD groups were insufficient for assessment by transmission electron microscopy. Therefore, transmission electron microscopy was done on samples obtained from seven children in each group. The median
(IQR) number of epithelial cells per sample studied was 380 (276664). Results are summarised in tables 2 and 3 (and tables S3 and S4 in the online supplementary material) and example electron micrographs are shown in figures 3 and 4 . Striking ultrastructural abnormalities were observed in the ciliated epithelium below the airway anastomosis, in both the CF and NSLD groups. Compared with the epithelium above, the airway anastomosis and that of the peripheral airway, the epithelium below the anastomosis in the CF group showed a significant reduction in the proportion of ciliated cells $(p<0.05)$ and a significant increase in the proportion of ciliated cells with loss of cilia $(p<0.05)$ and cilia with microtubular defects $(p<0.05)$. In addition, compared to the epithelium above the airway anastomosis, the epithelium below the anastomosis in the CF group showed a significant increase in the proportion of unciliated cells, dead cells, cells extruding from the cell surface, cells with cytoplasmic blebbing and cells with mitochondrial damage $(\mathrm{p}<0.01)$. Also, there was significantly higher ciliary disorientation in the epithelium below the airway anastomosis compared to that above the anastomosis, in the $\mathrm{CF}$ group $(\mathrm{p}<0.05)$.

TABLE 3 Analysis of epithelial ultrastructure of the non-suppurative lung disease group by transmission electron microscopy

\begin{tabular}{|c|c|c|c|}
\hline & Above anastomosis & Below anastomosis & Peripheral airway \\
\hline Ciliated cells & $83.2(79.9-84.9)^{\star *}$ & $40.6(30.9-59.3)$ & $59.6(53.6-68.5)$ \\
\hline Mucus cells & $4.7(3.5-5.6)^{*}$ & $8.5(7.1-11.4)$ & $3.4(2.7-4.7)^{*}$ \\
\hline Dead cells & $0^{\star *}$ & $12.4(9.9-15.9)$ & $1.8(0.6-9.7)$ \\
\hline Dynein arm defects & $2.3(1.9-3.6)$ & $5.4(2.9-8.1)$ & $3.3(2.4-9.5)$ \\
\hline Cells extruding from the surface & $16.3(5.1-18.6)^{\star *}$ & $49.2(37.1-58.4)$ & $41(14.9-81.3)$ \\
\hline Cells with cytoplasmic blebbing & $7.2(4.5-10.3)^{\star \star}$ & $45.3(41.6-48.3)$ & $27.9(15.3-41)$ \\
\hline Cells with mitochondrial damage & $6.4(4.3-9.0)^{\star *}$ & $26.6(17.9-36.5)$ & $10.2(9.3-48.1)$ \\
\hline Ciliary orientation degrees & $11.8(10.6-12.7)$ & $19.5(17.2-22.2)^{\#}$ & $23.6(20.3-26.8)^{\#}$ \\
\hline
\end{tabular}

Data presented as median percentage (interquartile range). *: $p<0.05 ;{ }^{* *}: \mathrm{p}<0.01$ when compared with epithelium below the airway anastomosis. ${ }^{*}: \mathrm{p}<0.05$ when compared with epithelium above the airway anastomosis. 

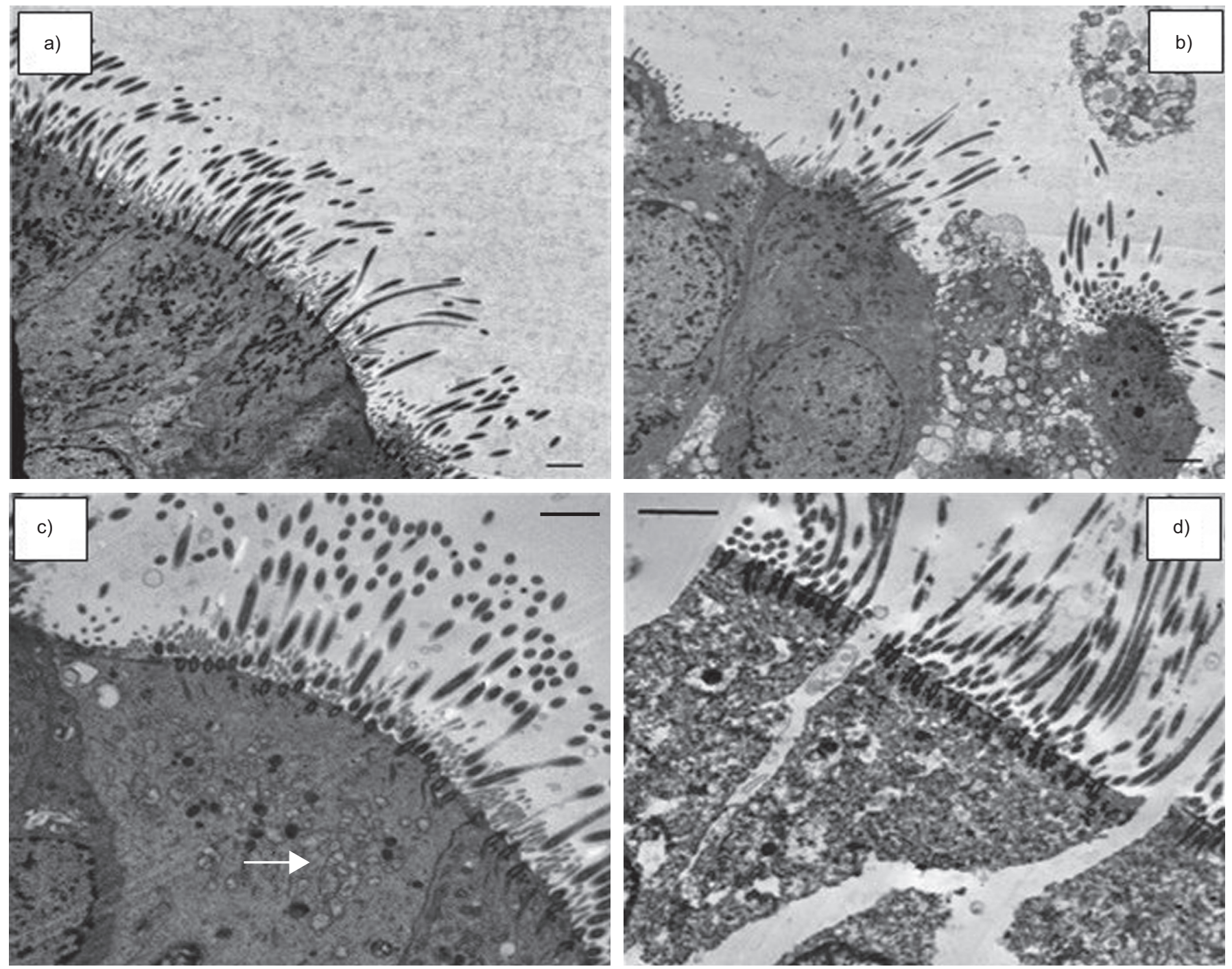

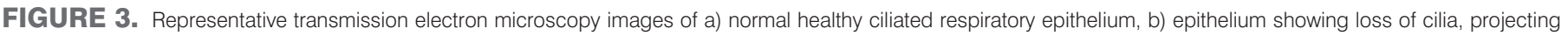
cell and disruption of epithelial tight junction with separation of cells, c) ciliated cell with disrupted and swollen mitochondria, as indicated by the arrow, and d) dead cells. Scale bars $=2 \mu \mathrm{m}$.

In the NSLD group, the epithelium below the airway anastomosis showed a significant reduction in the proportion of ciliated cells $(p<0.01)$ and a significant increase in the proportion of unciliated cells, dead cells, ciliated cells with loss of cilia, cells extruding from the cell surface, cells with cytoplasmic blebbing, cells with mitochondrial damage and cilia with microtubular defects $(p<0.01)$ when compared with the epithelium above the anastomosis. A significantly higher ciliary disorientation was seen in the epithelium below the airway anastomosis and the peripheral airway, when compared with the epithelium above the airway anastomosis $(p<0.05)$. The epithelium below the airway anastomosis also showed a significant increase in the proportion of mucus cells compared to that above the anastomosis and that of the peripheral airway $(\mathrm{p}<0.05)$.

In view of the possible effect of the normal epithelial healing process in the immediate post-operative period on epithelial ultrastructural changes, the data were re-analysed excluding the two patients (in each group) who had bronchial brushing samples taken within the first 4 months post transplantation. This did not alter the finding of significant epithelial ultrastructural abnormalities in the epithelium below the airway anastomosis (tables S3 and S4 in the online supplementary material). Re-analysis of the epithelial ultrastructural data excluding the subjects $(n=7)$ with $>10 \%$ lipid laden macrophages in the BAL also did not alter the finding of significant epithelial ultrastructural abnormalities in the epithelium below the airway anastomosis.

\section{DISCUSSION}

This study shows that, in paediatric CF lung transplant recipients, there is significant ciliary dysfunction in the epithelium above the airway anastomosis. However, the ciliary function of the native airway of paediatric NSLD lung-transplant recipients was comparable with that of the transplanted lungs. Our data suggests that there is no difference in the length and beat frequency of cilia from peripheral airways compared to that from central airways of the transplanted lungs. We have also 

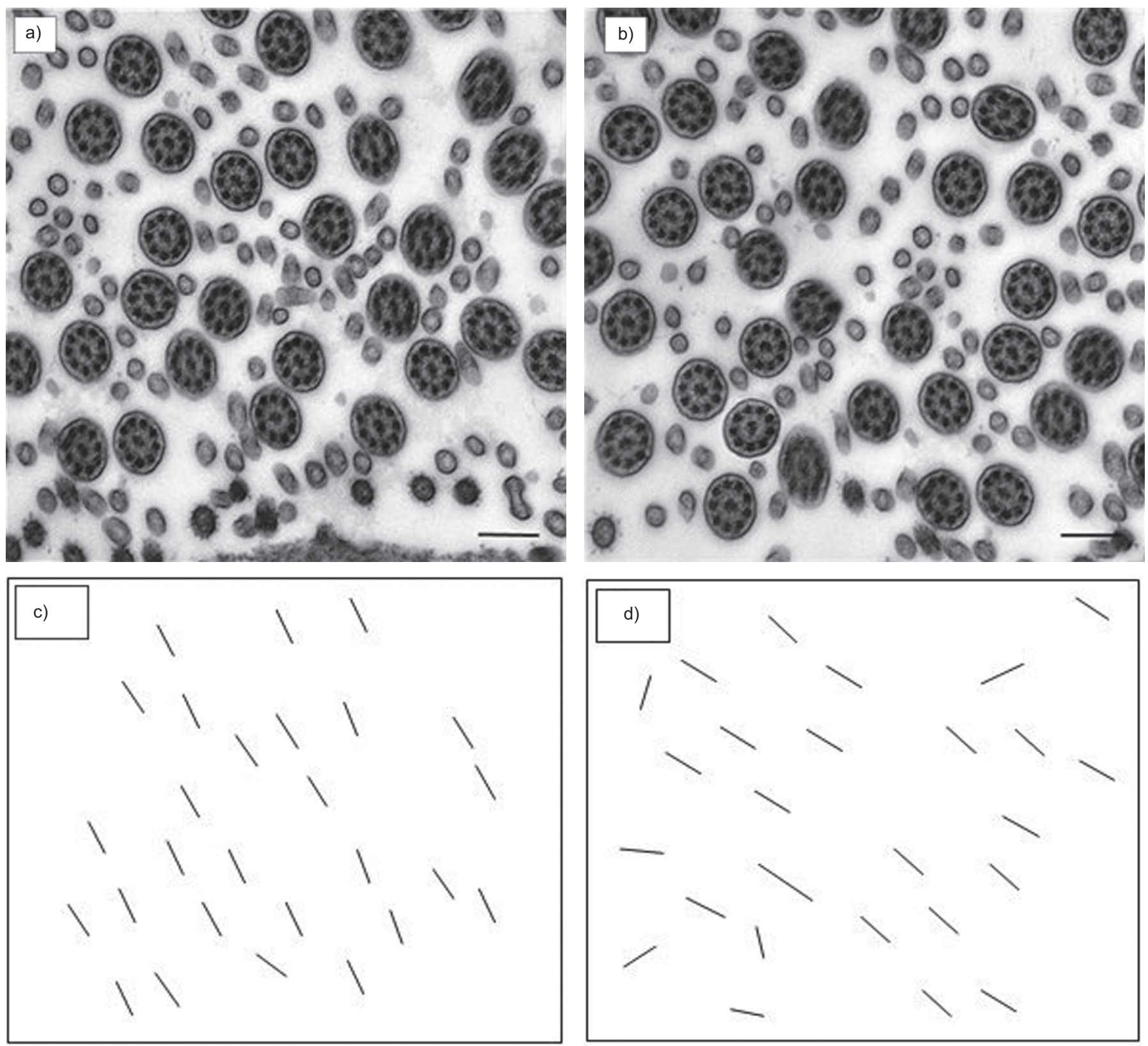

FIGURE 4. Cross sectional image of cilia originating from a) a normal healthy ciliated cell showing normal ciliary orientation (9.48 in this example) and b) a ciliated cell showing ciliary disorientation ( $35.12^{\circ}$ in this example). $\mathrm{c}$ and d) The distribution of lines drawn parallel to the central pair of microtubules of cilia originating from a single ciliated cell. Scale bars $=250 \mathrm{~nm}$.

shown that profound epithelial ultrastructural abnormalities persist in the epithelium below the airway anastomosis, for several months post transplantation, in both CF and NSLD paediatric lung transplant recipients. This is of concern as damage to the respiratory epithelium is known to predispose to secondary infection, a major problem in lung transplant patients.

To our knowledge this is the first study that assessed ciliary function (beat frequency and beat pattern) and epithelial ultrastructure in paediatric lung transplant recipients. Previous authors $[9,12,14]$ studied CBF in adult lung-transplant recipients and no difference in CBF between the native and transplanted bronchi were reported in all except one study [12]. Heterogeneity in the subject characteristics, indication for transplant, type of transplant and methodology used for sample collection and analysis of CBF makes direct comparison with our study results difficult. Our study is unique to any lung transplantation study in that we assessed the beat pattern in addition to measurement of CBF. This was made possible by the recent advent of highresolution digital high-speed video imaging [18], which enabled us to assess the precise beat pattern of cilia by viewing the ciliary beat cycle frame by frame in different planes. Using this method, we have previously shown that cilia in certain conditions may have a normal beat frequency despite a markedly abnormal beat pattern [20], and normal ciliary beat frequency does not necessarily equate to normal ciliary function. Our finding of 
significantly reduced beat frequency and increased ciliary dyskinesia in the epithelium of native airway in the CF transplant recipients is in contrast to the study by READ et al. [14] that showed a higher median (IQR) CBF of 10.8 (8.8-11.1) Hz in the native airway epithelium of the six adults with CF they studied. Though chronic inflammation, which is often present in the native CF airway epithelium, is known to cause ciliary dysfunction and ultrastructural defects [23], the precise mechanism(s) underlying ciliary dysfunction in the native CF epithelium remains to be determined. There has been a suggestion that compared to cilia in central airways, cilia in peripheral airways are shorter [24] and beat at a slower frequency [25, 26]. Our data contradicts this suggestion and is in agreement to the study by YAGER et al. [27] who showed no difference in CBF of epithelium from different levels of the tracheobronchial tree.

The findings of the limited animal and human studies that examined epithelial ultrastructure post lung transplantation, have been inconclusive [10,28]. In this study we systematically quantified the ultrastructural abnormalities of the respiratory epithelium of the native airway and transplanted lungs. Our findings of profound loss of epithelial integrity and striking ultrastructural abnormalities of the epithelium below the airway anastomosis, observed several months post transplantation, are likely to be multifactorial in causation. Injury during organ harvesting, allograft preservation, reperfusion and acute cellular rejection may cause epithelial damage. Although not studied in detail, devascularisation and lymphatic interruption due to transplantation, medications such as corticosteroids that may impair wound healing, and aspiration into airways due to gastro-oesophageal reflux [29] have all been implicated in epithelial damage. The relative preservation of epithelial integrity in the peripheral airways, compared to the airway just below the anastomosis, suggests possible effects of local mechanisms such as devascularisation and/or lymphatic interruption, though this remains to be determined.

The marked epithelial ultrastructural abnormalities that we observed below the airway anastomosis are very likely to have important functional consequences. First, these may result in quantitative and qualitative alterations in mucus and periciliary fluid, which may lead to impaired mucociliary clearance despite a normal CBF. Of particular interest, we observed increased ciliary disorientation in the epithelium of the transplanted lungs. A comparable degree of disorientation has been described previously as a variant of primary ciliary dyskinesia [21]. Secondly, and perhaps more importantly, these epithelial abnormalities may increase the risk of allograft colonisation by pathogenic organisms. Indeed, bacteria such as Pseudomonas have been shown to adhere preferentially to injured, disrupted and regenerating areas of airway epithelium [30] and colonise the allograft in both CF $[2,31,32]$ and non-CF [31, 32] lung transplant recipients. A growing body of evidence points towards the potential role of allograft colonisation with microbes such as Pseudomonas [2, 31, 32] and Aspergillus [5] in the development of BOS.

The main limitation of our study is its cross-sectional design; therefore, the within subject repeatability and longitudinal changes in ciliary function and epithelial ultrastructure need to be further studied. Our data generated several important questions that need to be addressed in future research. Unravelling the cause(s) of the ciliary dysfunction in the native
CF airway epithelium, epithelial ultrastructural abnormalities below the airway anastomosis and its potential effects on mucus, periciliary fluid and mucociliary clearance, pose major challenges for future studies. The suggestion in an animal model that preservation of peribronchial tissue during lung transplantation might potentially reduce the abnormalities in mucus rheology and airway epithelium [7], is of interest and future studies could assess the effect of different surgical techniques aimed at minimising effects of denervation, devascularisation and lymphatic obstruction, on allograft epithelial ultrastructure. Though previous studies [11, 28] suggested that the epithelial ultrastructural abnormalities post transplantation may recover as early as 3-4 months post-transplant, our data is not consistent with these observations and only long-term prospective studies will determine the time course of allograft epithelial ultrastructural changes post transplantation. Such information may guide therapeutic decisions, such as the duration of prophylactic antimicrobials. Despite the limitations of our study, our measures of ciliary function and epithelial cell morphology are highly repeatable and the magnitude of epithelial ultrastructural abnormalities seen below the airway anastomosis is striking, and is very likely to be clinically important.

In summary, our study provides evidence for ciliary dysfunction in the native airway of paediatric CF lung transplant recipients and marked epithelial damage below the airway anastomosis in both CF and NSLD paediatric lung transplant recipients. The potential major consequence of the damaged allograft epithelium is the increased risk of microbial colonisation of the allograft airway, which may play a role in the development of BOS.

\section{STATEMENT OF INTEREST}

None declared.

\section{ACKNOWLEDGEMENTS}

We are indebted to the children and their families who participated in this study; to the transplant unit specialist nurses at Great Ormond Street Hospital for Children (London, UK) for assistance in the clinical characterisation of the patients; G.M. Doherty (Great Ormond Street Hospital for Children) for helping with patient recruitment; J. Bankart for advice on statistical analysis and to M G. Williams and N Baker for technical assistance in the laboratory (all University of Leicester, Leicester, UK).

\section{REFERENCES}

1 Aurora P, Edwards LB, Kucheryavaya AY, et al. The Registry of the International Society for Heart and Lung Transplantation: thirteenth official pediatric lung and heart-lung transplantation report - 2010. J Heart Lung Transplant 2010; 29: 1129-1141.

2 Gottlieb J, Mattner F, Weissbrodt H, et al. Impact of graft colonization with Gram-negative bacteria after lung transplantation on the development of bronchiolitis obliterans syndrome in recipients with cystic fibrosis. Respir Med 2009; 103: 743-749.

3 Keenan RJ, Lega ME, Dummer JS, et al. Cytomegalovirus serologic status and postoperative infection correlated with risk of developing chronic rejection after pulmonary transplantation. Transplantation 1991; 51: 433-438.

4 Neurohr C, Huppmann P, Leuchte H, et al. Human herpesvirus 6 in bronchalveolar lavage fluid after lung transplantation: a risk factor for bronchiolitis obliterans syndrome? Am J Transplant 2005; 5: 2982-2991. 
5 Weigt SS, Elashoff RM, Huang C, et al. Aspergillus colonization of the lung allograft is a risk factor for bronchiolitis obliterans syndrome. Am J Transplant 2009; 9: 1903-1911.

6 Wanner A, Salathe M, O'Riordan TG. Mucociliary clearance in the airways. Am J Respir Crit Care Med 1996; 154: 1868-1902.

7 Paul A, Marelli D, Shennib H, et al. Mucociliary function in autotransplanted, allotransplanted, and sleeve resected lungs. J Thorac Cardiovasc Surg 1989; 98: 523-528.

8 Rivero DH, Lorenzi-Filho G, Pazetti R, et al. Effects of bronchial transection and reanastomosis on mucociliary system. Chest 2001; 119: 1510-1515.

9 Dolovich M, Rossman C, Chambers C, et al. Mucociliary function in patients following single lung or heart/lung transplantation. Am Rev Respir Dis 1987; 135: A363.

10 Sankar S, Fulsham L, Read R, et al. Mucociliary function after lung transplantation. Transplant Proceedings 1991; 23: 1222-1223.

11 Tomkiewicz RP, App EM, Shennib H, et al. Airway mucus and epithelial function in a canine model of single lung autotransplantation. Chest 1995; 107: 261-265.

12 Veale D, Glasper PN, Gascoigne A, et al. Ciliary beat frequency in transplanted lungs. Thorax 1993; 48: 629-631.

13 Nørgaard MA, Andersen CB, Pettersson G. Airway epithelium of transplanted lungs with and without direct bronchial artery revascularization. Eur J Cardiothorac Surg 1999; 15: 37-44.

14 Read RC, Shankar S, Rutman A, et al. Ciliary beat frequency and structure of recipient and donor epithelia following lung transplantation. Eur Respir J 1991; 4: 796-801.

15 Chilvers MA, Rutman A, O'Callaghan C. Ciliary beat pattern is associated with specific ultrastructural defects in primary ciliary dyskinesia. J Allergy Clin Immunol 2003; 112: 518-524.

16 Stannard W, Rutman A, Wallis C, et al. Central microtubular agenesis causing primary ciliary dyskinesia. Am J Respir Crit Care Med 2004; 169: 634-637.

17 Midulla F, de Blic J, Barbato A, et al. Flexible endoscopy of paediatric airways. Eur Respir J 2003; 22: 698-708.

18 Chilvers MA, O'Callaghan C. Analysis of ciliary beat pattern and beat frequency using digital high speed imaging: comparison with the photomultiplier and photodiode methods. Thorax 2000; 55: 314-317.
19 Greenstone M, Rutman A, Dewar A, et al. Primary ciliary dyskinesia: cytological and clinical features. Q J Med 1988; 67: 405-423.

20 Chilvers MA, McKean M, Rutman A, et al. The effects of coronavirus on human nasal ciliated respiratory epithelium. Eur Respir J 2001; 18: 965-970.

21 Bush A, Cole P, Hariri M, et al. Primary ciliary dyskinesia: diagnosis and standards of care. Eur Respir J 1998; 12: 982-988.

22 Seybold ZV, Mariassy AT, Stroh D, Kim CS, et al. Mucociliary interaction in vitro: effects of physiological and inflammatory stimuli. J Appl Physiol 1990; 68: 1421-1426.

23 Smallman LA. Microtubular abnormalities of cilia in acquired pulmonary diseases. Lancet 1984; 1: 965-966.

24 Serafini SM, Michaelson ED. Length and distribution of cilia in human and canine airways. Bull Eur Physiopathol Respir 1977; 13: 551-559.

25 Clary-Meinesz C, Mouroux J, Huitorel P, et al. Ciliary beat frequency in human bronchi and bronchioles. Chest 1997; 111: 692-697.

26 Rutland J, Griffin WM, Cole PJ. Human ciliary beat frequency in epithelium from intrathoracic and extrathoracic airways. Am Rev Respir Dis 1982; 125: 100-105.

27 Yager JA, Ellman H, Dulfano MJ. Human ciliary beat frequency at three levels of the tracheobronchial tree. Am Rev Respir Dis 1980; 121: 661-665.

28 Marelli D, Paul A, Nguyen DM, et al. The reversibility of impaired mucociliary function after lung transplantation. J Thorac Cardiovasc Surg 1991; 102: 908-912.

29 Effros RM, Jacobs ER, Schapira RM, et al. Response of the lungs to aspiration. Am J Med 2000; 108: Suppl. 4a, 15S-19S.

30 De Bentzmann S, Plotkowski M, Puchelle E. Receptors in the Pseudomonas aeruginosa adherence to injured and repairing airway epithelium. Am J Respir Crit Care Med 1996; 154: S155-S162.

31 Botha P, Archer L, Anderson RL, et al. Pseudomonas aeruginosa colonization of the allograft after lung transplantation and the risk of bronchiolitis obliterans syndrome. Transplantation 2008; 85: 771-774.

32 Vos R, Vanaudenaerde BM, Geudens N, et al. Pseudomonal airway colonisation: risk factor for bronchiolitis obliterans syndrome after lung transplantation? Eur Respir J 2008; 31: 1037-1045. 